\title{
ON THE DIMENSION OF MODULES
}

AND ALGEBRAS, II

\section{(FROBENIUS ALGEBRAS AND QUASI-FROBENIUS RINGS)}

\author{
SAMUEL EILENBERG* and TADASI NAKAYAMA
}

In this paper we study Frobenius algebras and quasi-Frobenius rings with particular emphasis on their cohomological dimensions. For definitions of these cohomological dimensions we refer the reader to Cartan-Eilenberg [3] or Eilenberg [4].

We consider (in §2) symmetric and Frobenius algebras in a setting more general than that of Brauer and Nesbitt [2], [13], and show (in §3) that for such algebras the cohomological dimension is either 0 or $\infty$.

These results are applied $(\S 4)$ to the group ring $A=K(I I)$ where $I I$ is a finite group of order $r$ and $K$ is a commutative ring. It is shown that $A$ is symmetric and that $\operatorname{dim} A=0$ if $r K=K$ and that $\operatorname{dim} A=\infty$ if $r K \neq K$.

The phenomenon that the cohomological dimension is either 0 or $\infty$ is again encountered $(\S 5)$ in a ring $A$ which is left self-injective i.e. a ring $\Lambda$ which when regarded as a left $\Lambda$-module is injective. Such rings, under different terminologies have been considered recently in Ikeda [7], Nagao-Nakayama [10] and Ikeda-Nakayama [9] in connection with quasi-Frobenins algebras and rings. We further refine these results by showing $(\S \S 6,7)$ that the notions "quasi-Frobenius ring" and "left self-injective ring" are equivalent for rings which are (left and right) Noetherian, or satisfy minimum condition for left or right ideals.

All rings considered have a unit element which operates as the identity on all modules considered.

\section{§ 1. Duality}

Let $K$ be a commutative ring. For each $K$-module $A$ we define the dual $K$-module

$$
A^{\circ}=\operatorname{Hom}_{\kappa}(A, K) \text {. }
$$

Received June 24, 1954.

* This work was done while S. Eilenberg was engaged under contract AF-18(600)-562. 
A $K$-homomorphism $f: A \rightarrow B$ induces a dual homomorphism $f^{\circ}: B^{\circ} \rightarrow A^{\circ}$, in the well-known natural fashion.

Given $K$-modules $A$ and $C$ we define

$$
\tau: A \otimes_{K} C \rightarrow \operatorname{Hom}_{K}\left(A^{\circ}, C\right)
$$

by setting

$$
[\tau(a \otimes c)] f=(f a) c, \quad a \in A, \quad c \in C, \quad f \in A^{\circ} .
$$

Clearly $\tau$ is an isomorphism if $A=K$. Therefore, by a simple direct sum argument it follows that $\tau$ is also an isomorphism if $A$ is $K$-projective and finitely $K$-generated.

Taking $C=K$ we obtain a natural homomorphism

$$
\tau: A \rightarrow A^{\circ \circ}
$$

which is again an isomorphism if $A$ is $K$-projective and finitely $K$-generated.

For each $K$-homomorphism

$$
\varphi: A \rightarrow C^{\circ}
$$

the transposed homomorphism

$$
\varphi^{\prime}: C \rightarrow A^{\circ}
$$

is defined by composition

$$
C \stackrel{\tau}{\longrightarrow} C^{\circ \circ} \stackrel{\Phi^{\circ}}{\longrightarrow} A^{\circ}
$$

It is easily seen that

$$
\left(\varphi^{\prime} c\right) a=(\varphi a) c .
$$

This shows that $\varphi^{\prime \prime}=\varphi$. If $C$ is $K$-projective and finitely $K$-generated and if $\varphi$ is an isomorphism then $\varphi^{\prime}$ is an isomorphism.

Now assume that $A$ is a $K$-algebra and that $A$ is a left (right) $\Lambda$-module. Then $A^{\circ}$ is a right (left) $A$-module and it follows from the naturality of $\tau$ that $\tau$ is a $A$-homomorphism. If $A$ is a left $A$-module, $C$ is a right $A$-module and $\varphi$ above is a $\Lambda$-homomorphism then $\varphi^{\prime}$ also is a $A$-homomorphism.

As usual we shall regard $\Lambda$ as a two-sided $A$-module and consequently $\Lambda^{\circ}$ is also a two-sided $A$-module. It should however be noted that in defining the structure of $\Lambda^{\circ}$ as a left (right) $A$-module we utilize the structure of $A$ as a right (left) $A$-module. 


\section{§. Frobenius algebras}

Definition Let $A$ be a $K$-algebra which is $K$-projective and finitely $K$ generated. We shall say that $A$ is a Frobenius algebra if there exists a left $\Lambda^{-}$ module isomorphism

$$
\Phi: \Lambda \approx \Lambda^{\circ}
$$

If there exists a two-sided $\Lambda$-module isomorphism $\Phi: \Lambda \approx \Lambda^{\circ}$ then $\Lambda$ is called a symmetric algebra.

Starting with a left $\Lambda$-module isomorphism $\Phi$ as above we obtain a right A-module isomorphism

$$
\mathscr{D}^{\prime}: \Lambda \approx \Lambda^{\circ}
$$

by taking the transposed

$$
\left[\Phi^{\prime} \lambda\right](\gamma)=\left[\Phi_{\gamma}\right](\lambda), \quad \lambda, \gamma \in \Lambda .
$$

Since $\Phi \lambda=\lambda \Phi 1$ and $\Phi^{\prime} \lambda=\left(\Phi^{\prime} 1\right) \lambda$ it follows readily that

$$
\left[\Phi_{\gamma}\right](\lambda)=\varphi(\lambda \gamma)=\left[\Phi^{\prime} \lambda\right](\gamma)
$$

where

$$
\varphi=\emptyset 1=\Phi^{\prime} 1: \Lambda \rightarrow K .
$$

Conversely we may begin with a $K$-homomorphism $\varphi: \Lambda \rightarrow K$ and define $\Phi$ (and $\left.\Phi^{\prime}\right)$ using (*). Then $\Phi$ is a left $\Lambda$-homomorphism $\Lambda \rightarrow \Lambda^{\circ}$. The conditions that $\Phi$ be a monomorphism and epimorphism are respectively

$$
\varphi(\lambda \gamma)=0 \text { for all } \lambda \in \Lambda \text { implies } \gamma=0,
$$

$$
\text { for each } f \in \Lambda^{\circ} \text {, there is a } r \in \Lambda \text { such that } f \lambda=\varphi(\lambda \gamma) \text {. }
$$

Applying the same reasoning to $\Phi^{\prime}$ we find the conditions

$$
\varphi(\lambda \gamma)=0 \text { for all } \gamma \in \Lambda \text { implies } \lambda=0,
$$

(r.2) for each $f \in \Lambda^{\circ}$ there is a $\lambda \in \Lambda$ such that $f \gamma=\varphi(\lambda r)$.

The two sets of conditions are thus equivalent.

If $\Lambda$ is symmetric and $\Phi: \Lambda \approx \Lambda^{\circ}$ is a two-sided $\Lambda$-isomorphism, then the relation $\Phi 1=\Phi^{\prime} 1$ implies $\Phi=\Phi^{\prime}$, or equivalently

$$
\varphi(\lambda r)=\varphi(\gamma \lambda) \text {. }
$$

Conversely if $\varphi$ satisfies this condition (in addition to conditions (1.1), (1.2) or 
(r. 1 ), (r.2)) then $\Phi=\Phi^{\prime}$ is a two-sided isomorphism $\Lambda \approx \Lambda^{\circ}$, and $\Lambda$ is symmetric.

Remark. Condition (1.1) asserts that the "hyperplane" in $A$ given by $\varphi=0$ does not contain any left ideals except zero.

Remark. If $K$ is a field, then $A$ and $A^{\circ}$ are vector spaces of the same finite degree over $K$. Thus if $\Phi$ is a monomorphism, it is necessarily an isomorphism. Consequently in this case condition (1.2) is a consequence of (1.1). Similarly (r.2) follows from (r.1). Conditions (1.1) and (r.1) are then equivalent.

Proposition 1. Let $\Lambda_{1}, A_{2}$ be $K$-algebras and $A=A_{1}+A_{2}$ their direct product. Then $\Lambda$ is a Frobenius (or a symmetric) algebra if and only if the same holds for $\Lambda_{1}$ and $\Lambda_{2}$.

Proof. Clearly $A$ is $K$-projective and finitely $K$-generated if and only if $\Lambda_{1}$ and $A_{2}$ are. The rest follows from the isomorphism $\left(A_{1}+A_{2}\right)^{\circ} \approx A_{1}^{\circ}+A_{2}^{\circ}$ for the direct sum of any $K$-modules $A_{1}$ and $A_{2}$.

Proposition 2. If $\Lambda_{1}$ and $\Lambda_{2}$ are Frobenius (or symmetric) $K$-algebras, then so is $A=A_{1} \otimes_{K} \Lambda_{2}$.

Proof. Clearly $A$ is $K$-projective and finitely $K$-generated.

For any $K$-modules $A_{1}$ and $A_{2}$ consider the mapping

$$
\zeta: A_{1}^{\circ} \otimes_{K} A_{2}^{\circ} \rightarrow\left(A_{1} \otimes_{K} A_{2}\right)^{\circ}
$$

given by

$$
\left[\zeta\left(f_{1} \otimes f_{2}\right)\right]\left(a_{1} \otimes a_{2}\right)=f_{1} a_{1} \otimes f_{2} a_{2} .
$$

Clearly $\zeta$ is an isomorphism for $A_{1}=A_{2}=K$. It follows that $\zeta$ is an isomorphism also if $A_{1}$ and $A_{2}$ are $K$-projective and finitely $K$-generated. Thus

$$
\zeta: \Lambda^{\circ} \approx \Lambda_{1}^{\circ} \otimes_{K} \Lambda_{2}^{\circ}
$$

and, since $\zeta$ is natural, this is an isomorphism of two-sided 1 -modules. This yields the conclusion.

Proposition 3. A full matrix algebra $A$ over a commutative ring $K$ is symmetric.

Proof. The $K$-homomorphism $A \rightarrow K$ given by the trace is easily seen to satisfy (1.1), (1.2) and (s). 
Proposition 4. A division algebra $\Lambda$ over a field $K$ with $(\Lambda: K)<\infty$ is symmetric.

Proof. For each $K$-algebra $\Lambda$ let $[\Lambda, \Lambda]$ denote the subgroup generated by the commutators $\lambda \gamma-\gamma \lambda$, for $\lambda, \gamma \in \Lambda$. Automatically $[A, \Lambda]$ is a $K$-module.

Assume now that $\Lambda$ is a division algebra over the field $K$ with $(\Lambda: K)<\infty$. Any non-zero $K$-homomorphism $\varphi: \Lambda \rightarrow K$ satisfies (1.1), so that $\Lambda$ is a Frobenius algebra. In order that $\varphi$ satisfy also condition (s) it is necessary and sufficient that $\varphi[A, A]=0$. Thus it suffices to prove that $[A, \Lambda] \neq \Lambda$. This last statement being independent of the ground-field we may assume that $K$ is the center of A. Let $L$ be an extension of $K$ which is a splitting field for $\Lambda$. Then the $L$-algebra $\Lambda_{L}=\Lambda \otimes_{K} L$ is a full matrix algebra over $L$ and thus, by Prop. 3, $\Lambda_{L}$ is a symmetric $L$-algebra. It follows that $\left[\Lambda_{L}, \Lambda_{L}\right] \neq \Lambda_{L}$. Since $\left[\Lambda_{L}, \Lambda_{L}\right]$ $=[\Lambda, \Lambda]_{L}$, we deduce that $[\Lambda, \Lambda] \neq \Lambda$.

Combining Prop. 1, 2, 3 and 4 we obtain (cf. [11], footnote 51):

Proposition 5. A semi-simple algebra $\Lambda$ over a field $K$ with $(\Lambda: K)<\infty$ is symmetric.

Proposition 6. Let $A$ be an algebra over a field $K$ with $(A: K)<\infty$, and let $L$ be an extension field of $K$. The algebra $A_{L}=\Lambda \otimes_{K} L$ over $L$ is a Frobenius (or a symmetric) algebra if and only if $\Lambda$ is so.

Proof. This follows easily from our definition of Frobenius and symmetric algebras and the fact (E. Noether's lemma) that two (left, right or two-sided) $\Lambda$-modules $A_{1}, A_{2}$ with $\left(A_{1}: K\right)<\infty,\left(A_{2}: K\right)<\infty$ are isomorphic if and only if the $A_{L}$-modules $\left(A_{1}\right)_{L}=A_{1} \otimes{ }_{K} L$ and $\left(A_{2}\right)_{L}=A_{2} \otimes{ }_{K} L$ are isomorphic (cf. e.g. M. Deuring, Galoissche Theorie und Darstellungstheorie, Math. Ann. 107 (1933), p. 144).

\section{§3. Dimension in Frobenius algebras}

Proposition 7. If $\Lambda$ is a Frobenius algebra, over a commutative ring $K$, then we have a natural isomorphism

$$
\operatorname{Ext}_{\Lambda}^{q}\left(A, A \otimes{ }_{K} C\right) \approx \operatorname{Ext}_{K}^{q}(A, C)
$$

for each left $A$-module $A$ and each $K$-module $C$.

Proof. Assuming a fixed isomorphism $\Phi^{\prime}: \Lambda \approx \Lambda^{\circ}$ (of right $\Lambda$-modules) and 
utilizing the map $\tau$ we have

$$
\begin{aligned}
\operatorname{Hom}_{\Lambda}\left(A, A \otimes \otimes_{K} C\right) & \approx \operatorname{Hom}_{\Lambda}\left(A, \operatorname{Hom}_{K}\left(\Lambda^{\circ}, C\right)\right) \approx \operatorname{Hom}_{\Lambda}\left(A, \operatorname{Hom}_{K}(A, C)\right) \\
& \approx \operatorname{Hom}_{K}\left(A \otimes \otimes_{\Lambda} A, C\right) \approx \operatorname{Hom}_{K}(A, C) .
\end{aligned}
$$

This gives the desired isomorphism (3.1) for $q=0$. Now replace $A$ by a $A$ projective resolution $X$. Since $A$ is $K$-projective, $X$ also is a $K$-projective resolution of $A$. Passing to homology yields (3.1) in virtue of the definitions of Ext $_{\Lambda}$ and Ext $_{K}$.

Remark. The isomorphism (3.1) although "natural" is not necessarily unique, as it depends upon the choice of $\Phi^{\prime}$. With $\Phi^{\prime}: \Lambda \approx \Lambda^{\circ}$ fixed, the isomorphisms (3.1) obtained, commute properly with maps induced by $A$-homomorphisms of $A$ and $K$-homomorphisms of $C$.

Corollary 8. If $A$ is a Frobenius $K$-algebra and $C$ is a $K$-module, then 1.inj. $\operatorname{dim}_{\Lambda}\left(\Lambda \otimes{ }_{K} C\right) \leqq$ inj. $\operatorname{dim}_{K} C$.

Corollary 9. If $A$ is a Frobenius algebra and $K$ is self-injective then $A$ is both left and right self-injective.

Theorem 10. If $\Lambda$ is a Frobenius K-algebra and $A$ is a left A-module satisfying l. $\operatorname{dim}_{\mathrm{\Lambda}} A<\infty$, then

$$
\text { 1. } \operatorname{dim}_{\Delta} A=1 . \operatorname{dim}_{K} A \text {. }
$$

Proof. Let $1 \operatorname{dim}_{\Lambda} A=n<\infty$, and let $C$ be a left $A$-module such that $\operatorname{Ext}_{\Lambda}^{n}(A, C) \neq 0$. Consider an exact sequence $0 \rightarrow B \rightarrow F \rightarrow C \rightarrow 0$ with $F A$-free. Since $\operatorname{Ext}_{\Lambda}^{n+1}(A, B)=0$, it follows from exactness that $\operatorname{Ext}_{\Lambda}^{n}(A, F) \rightarrow \operatorname{Ext}_{\Lambda}^{n}(A, C)$ is an epimorphism and therefore $\operatorname{Ext}_{\Lambda}^{n}(A, F) \neq 0$. Since $F \approx A \otimes_{K} H$ where $H$ is a free $K$-module, it follows from Prop. 7 that $\operatorname{Ext}_{K}^{n}(A, H) \neq 0$. Thus $1 . \operatorname{dim}_{\mathrm{\Lambda}} A \leqq \operatorname{dim}_{K} A$. The opposite inequality is trivial since $A$ is $K$-projective.

TheOREM 11. If $\Lambda$ is a Frobenins K-algebra then $\operatorname{dim} \Lambda=0, \infty$.

Proof. Clearly $\Lambda^{*}$ (the algebra opposite to $\Lambda$ ) also is a Frobenius $K$-algebra. Thus it follows from Prop. 2 that $\Lambda^{e}=\Lambda \otimes_{K} A^{*}$ also is a Frobenius $K$-algebra. Consequently, if $\operatorname{dim} \Lambda<\infty$, we have, by Theorem 10 ,

$$
\operatorname{dim} \Lambda=1 . \operatorname{dim}_{\Lambda^{e}} \Lambda=1 . \operatorname{dim}_{K} \Lambda=0 .
$$

The special case of Theorem 11 with $K$ a field has been established in [8] 
( $\$$, Corollary); cf. also Corollary 21 (and Corollary 19) below. A further particular case is a result of Hochschild [6] (see also [4]) that if $\Lambda$ is a semisimple algebra over a field $K$ with $(A: K)<\infty$ then either $A$ is separable (i.e. $\operatorname{dim} A=0$ ) or $\operatorname{dim} \Lambda=\infty$.

\section{$\S 4$. The algebra of a finite group}

Let $\Pi$ be a finite group of order $r$ and let $K$ be a commutative ring. The group algebra $\Lambda=K(I I)$ is defined as usual. This is a supplemented algebra under the map $\varepsilon: A \rightarrow K$ given by $\varepsilon x=1$ for all $x \in \Pi$. Using this map, we may regard $K$ as a (right or left) $A$-module.

We first show that $\Lambda=K(\Pi)$ is symmetric. To this end we define a $K$ homomorphism $\varphi: A \rightarrow K$ by setting $\varphi 1=1, \varphi x=0$ for $x \in \Pi, x \neq 1$. If $\gamma$ $=\sum_{x \in 11} a(x) x$ for $a(x) \in K$, then $\varphi\left(x^{-1} \gamma\right)=a(x)$. Thus $\varphi\left(\lambda_{r}\right)=0$ for all $\lambda \in \Lambda$ implies $\gamma=0$, so that (1.1) is satisfied. Next let $f: A \rightarrow K$ be a $K$-homomorphism and let $\gamma=\sum f(x) x^{-1}, x \in \Pi$. Then for $y \in \Pi$

$$
\varphi(y \gamma)=\varphi\left(\sum f(x) y x^{-1}\right)=f(y)
$$

so that (1.2) also is satisfied. Finally condition (s) holds trivially. Thus $\Lambda$ is symmetric.

Note that the map 9 defined above yields an isomorphism and in consequence, as in the proof of Prop. 7 yields an isomorphism

$$
\rho: \operatorname{Hom}_{K}(A, C) \approx \operatorname{Hom}_{\Lambda}\left(A, \Lambda \otimes{ }_{K} C\right)
$$

for a left $A$-module $A$ and a $K$-module $C$. This isomorphism $\rho$ may be written directly as

$$
(\rho f) a=\sum_{x \in 11} x \otimes f\left(x^{-1} a\right), \quad f \in \operatorname{Hom}_{K}(A, C), \quad a \in A .
$$

We may now apply the results of the preceding section. First we observe that since each $K$-module may also be regarded as a $\Lambda$-module (using $\varepsilon: \Lambda \rightarrow K$ ), Corollaries 8 and 9 may be strengthened as follows

Corollary 8 . 1. inj. $\operatorname{dim}_{\Lambda}\left(A \otimes{ }_{K} C\right)=1$. inj. $\operatorname{dim}_{K} C$

Corollary $9^{\prime} . A$ is left (or right) self-injective if and only $K$ is self-injective.

THEOREM 12. If $r K=K$ (where $r$ is the order of the group $I$ ), then 


$$
\operatorname{dim} A=l . \operatorname{dim}_{\Lambda} K=0 .
$$

If $r K \neq K$, then

$$
\operatorname{dim} \Lambda=l . \operatorname{dim}_{\Lambda} K=\infty .
$$

Proof. The equality $\operatorname{dim} A=1 \cdot \operatorname{dim}_{\mathrm{A}} K$ has been proved in [3] (Ch. $X, \S 6$ ) without any case subdivision and is valid also for infinite groups $I$. Since $\operatorname{dim}_{K} K=0$ it follows from Theorem 10 that $1 . \operatorname{dim}_{\Lambda} K$ is either 0 or $\infty$. Thus everything reduces to the question as to when $K$ is $A$-projective. Clearly this is the case if and only if there exists a $A$-homomorphism $\psi: K \rightarrow A$, such that $\varepsilon \psi=$ identity. Such a $\psi$ must satisfy $\phi 1=\sum_{x \in 11} \alpha x$ for some $\alpha \in K$. The condition $\varepsilon \psi 1=1$ then yields $x \alpha=1$. The existence of $\psi$ is thus equivalent with $r K=K$.

Remark. The above argument shows that if $I$ is an infinite group then $K$ is not $A$-projective.

Remark. The proof above utilized the equality $\operatorname{dim} A=1 . \operatorname{dim}_{\mathrm{\Delta}} K$ established by general methods. Actually, in the case considered here an ad hoc argument can be applied. Indeed, since $A$ is symmetric, it follows from Theorem 11 that $\operatorname{dim} A=0, \infty$. This we must find out when $\operatorname{dim} A=0$ i.e. when $A$ is $A^{e}$-projective, where $\Lambda^{e}=\Lambda \otimes_{K} \Lambda^{*}$. We have the $\Lambda^{e}$-epimorphism $\eta_{y}: A^{e} \rightarrow A$ given by $\eta\left(x \otimes y^{*}\right)$ $=x y, x, y \in \Pi$. Thus $A$ is $\Lambda^{e}$-projective if and only if there is a $A^{e}$-homomorphism $\psi: \Lambda \rightarrow A^{e}$ with $\eta \psi=$ identity. The map $\psi$ has the form

$$
\phi(x)=\sum_{y, z} k(x, y, z) y \otimes z^{*} \quad k(x, y, z) \in K
$$

with summation extended over all $y, z \in \Pi$. The condition that $\psi$ is a $A^{e}$ homomorphism becomes

$$
k(x z, y, 1)=k(x, y, z)=k(y x, 1, z)
$$

so that setting $\gamma(x)=k(x, 1,1)$ we have

$$
\psi(x)=\sum_{y, z} r(y x z) y \otimes z^{*} .
$$

Conversely any map $\gamma: \Pi \rightarrow K$ defines a $A^{e}$-homomorphism $\psi$. Then

$$
\eta \psi(1)=\sum_{y, z} \gamma(y z) y z=r \sum_{x} \gamma(x) x
$$


and the condition $\eta \psi=$ identity is equivalent with

$$
r(1)=1, \quad \gamma(x)=0 \quad \text { for } x \neq 1 \text {. }
$$

Hence $A$ is $A^{e}$-projective if and only if $r K=K$.

As a corollary of Theorem 12 we obtain the following known result (see [6], p. 948) :

Corollary 13. Let $K$ be a field of characteristic $p$. If $p=0$ or $(p, r)=1$ then $A=K(\Pi)$ is separable. If $(p, r) \neq 1$ then $\operatorname{dim} A=1 \cdot \operatorname{dim}_{\Lambda} K=\infty$.

In the case $r K=K$ we have a more detailed result which may be of interest.

Proposition 14. If $r K=K$ then for each pair of left $A$-modules $A$ and $C$ the $K$-module $\operatorname{Ext}_{\Lambda}^{q}(A, C)$ is isomorphic with a direct summand of $\operatorname{Ext}_{\Lambda}^{q}(A, C)$. This implies

$$
\begin{aligned}
\text { 1. } \operatorname{dim}_{\Lambda} A & =\operatorname{dim}_{K} A, \\
\text { 1. inj. } \operatorname{dim}_{\Lambda} C & \leqq \text { inj. } \operatorname{dim}_{K} C, \\
\text { 1. gl. } \operatorname{dim} A & =\text { gl. } \operatorname{dim} K .
\end{aligned}
$$

Proof. Let $\alpha \in K$ be such that $r \alpha=1$. Consider the homomorphisms

$$
\operatorname{Hom}_{\Lambda}(A, C) \stackrel{j}{\longrightarrow} \operatorname{Hom}_{K}(A, C) \stackrel{i}{\longrightarrow} \operatorname{Hom}_{\Lambda}(A, C)
$$

where $i$ is the inclusion, while

$$
\text { (jf) } a=\alpha \sum_{x} x f\left(x^{-1} a\right) .
$$

If $f$ is a $A$-homomorphisms then $x f\left(x^{-1} a\right)=f(a)$ and $j f=f$. Thus $j i=$ identity. Now replace $A$ by a projective resolution $X$ of $A$ and pass to homology. There result homomorphisms

$$
\operatorname{Ext}_{\Lambda}^{q}(A, C) \longrightarrow \operatorname{Ext}_{K}^{q}(A, C) \longrightarrow \operatorname{Ext}_{\Lambda}^{q}(A, C)
$$

whose composition is the identity. This yields the desired conclusion.

Incidentally, the case $r K=K$ with $K$ not semi-simple gives an example of an algebra $A=K(I I)$ with $\operatorname{dim} A=0$ and 1.gl. $\operatorname{dim} A=g l \cdot \operatorname{dim} K>0$.

\section{$\S 5$. Self-injective rings}

Let again $K$ be a commutative ring.

THEOREM 15. Let $\Lambda$ be a K-algebra which is $K$-projective, finitely $K$-generated 
and left self-injective. Then each $K$-projective and finitely $K$-generated left $A$ module $A$ such that l. $_{\text {imm }} A<\infty$ is projective and injective.

Proof. Since $A$ is left self-injective, every free (left) $A$-module on a finite base is injective. Therefore every finitely generated projective left $d$-module is injective.

Assume $1 . \operatorname{dim}_{\Lambda} A=n, 0<n<\infty$ and assume the result already proved for $n-1$. Consider the exact sequence

$$
0 \longrightarrow B \longrightarrow \Lambda \otimes_{K} A \stackrel{\alpha}{\longrightarrow} A \longrightarrow 0
$$

where $\alpha(\lambda \otimes a)=\lambda a, B=\operatorname{Ker} \alpha$. Since $A$ is $K$-projective, $A \otimes{ }_{K} A$ is $A$-projective and therefore $1 . \operatorname{dim}_{\mathrm{\Lambda}} B=n-1$. The $K$-homomorphism $\zeta: A \rightarrow \Lambda \otimes{ }_{\mathrm{K}} A$ given by $\zeta a=1 \otimes a$ satisfies $\alpha \zeta=$ identity and shows that the exact sequence splits over $K$. Since both $A$ and $A$ are $K$-projective and finitely $K$-generated, the same is true for $A \otimes_{K} A$ and therefore also for $B$ which is a $K$-direct summand of $A \otimes_{K} A$. Thus by the inductive assumption $B$ is $A$-projective and $A$-injective. Therefore the exact sequence splits (over $A !$ ) and thus $A$ is $A$-projective, and hence also $A$-injective.

Theorem 16. Let $A$ be a left Noetherian ring (i.e. a ring satisfying maximum condition for left ideals) which is left self-injective. Then each left $A^{-}$ module $A$ such that l. $\operatorname{dim}_{\mathrm{\Lambda}} A<\infty$ is projective and injective. In particular l.gl. $\operatorname{dim} \Lambda=0, \infty$.

Proof. Since $\Lambda$ is left Noetherian the direct sum of injective left $\Lambda$-modules is injective (see [3], Ch. I, Exer. 8). Therefore every free $A$-module is injective and consequently every projective left $A$-module is injective.

Now let $1 \operatorname{dim}_{\mathrm{\Lambda}} A=n<\infty$. As in the proof of Theorem 10 we have $\operatorname{Ext}_{\Lambda}^{n}(A, F)=0$ for some free $A$-module $F$. Since $F$ is injective it follows that $n=0$ and $A$ is projective.

Proposition 17. Let $A$ be a left Noetherian, left self-injective and nonsemisimple ring. If $A$ is an algebra over a semisimple commutative ring $K$ then $\operatorname{dim} \Lambda=\infty$.

Proof. Since $K$ is semisimple we have $1 . \operatorname{gl} . \operatorname{dim} A \leqq \operatorname{dim} A$ (see [3] or [4]), and by the preceding theorem 1.gl. $\operatorname{dim} 1=\infty$. 
Proposition 18. Let $A$ be a left Noetherian ring which is left self-injective. Then for a left ideal $\mathfrak{l}$ of $A$ the following conditions are equivalent :

(i) $1 . \operatorname{dim}_{\mathrm{A}} A / \mathfrak{l}<\infty$,

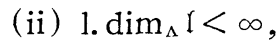

(iii) $\mathfrak{l}$ is projective,

(iv) $\mathfrak{l}$ is injective,

(v) $\mathfrak{l}=A e$ with an idempotent elemnt $e$ in $A$,

(vi) $A / \mathfrak{I}$ is projective.

Proof. The equivalences (i) $\Leftrightarrow(v i)$ and (ii) $\Leftrightarrow$ (iii) follow from Theorem 16. Thus it suffices to establish the equivalence of (iii), (iv), (v) and (vi).

(iii) $\Rightarrow$ (iv) follows from Theorem 16 .

(iv) $\Rightarrow(\mathrm{v})$. Since $\mathfrak{l}$ is injective there exists a projection $p: A \rightarrow \mathfrak{l}, p \lambda=\lambda$ for $\lambda \in \mathfrak{l}$. Then $\lambda=p(\lambda)=p(\lambda 1)=\lambda p(1)$ for $\lambda \in \mathfrak{l}$. Thus $e=p 1$ is idempotent and $\mathfrak{l}=\Lambda e$.

$(\mathrm{v}) \Rightarrow(\mathrm{vi})$. If $\mathfrak{l}=A e$ then $\Lambda / \mathfrak{l} \approx \Lambda(1-e)$ and thus $A / \mathfrak{l}$ is isomorphic to a direct summand of $\Lambda$.

(vi) $\Rightarrow$ (iii) follows from the exact sequence $0 \rightarrow \mathfrak{l} \rightarrow A \rightarrow A / \mathfrak{l} \rightarrow 0$ which splits if $\Lambda / \mathfrak{l}$ is projective.

\section{§6. Quasi-Frobenius rings}

For each subset $X$ of a ring $A$ we denote by $l(X)$ (or $r(X)$ ) the set of all left (or right) annihilators of $X$. Clearly $l(X)$ is a left ideal while $r(X)$ is a right ideal. If $X$ is a two-sided ideal then so are $l(X)$ and $r(X)$.

Quasi-Frobenius rings were defined by Nakayama [12] p. 8. For the purposes of this paper we adopt the following alternative definition ([12], p. 9, Th. 6 ) : $A$ ring $A$ is a quasi-Frobenius ring if it satisfies minimum conditions for left and right ideals and if the relations

$$
l(r(\mathfrak{l}))=\mathfrak{l}, \quad r(l(\mathfrak{r}))=\mathfrak{r}
$$

hold for all left ideals $\mathfrak{l}$ and all right ideals $\mathfrak{r}$.

Actually, in the presence of $(\alpha)$ it suffices to assume that $A$ is left (or right) Noetherian, i.e. that $\Lambda$ satisfies maximum condition for left (or right) ideals. The minimum conditions for both left and right ideals then follow.

THEOREM 18. For each ring 1 the following conditions are equivalent: 
(i) $A$ is a quasi-Frobenius ring.

(ii) $A$ is left Noetherian and the relations

$$
r\left(\mathfrak{l}_{1} \cap \mathfrak{I}_{2}\right)=r\left(\mathfrak{I}_{1}\right)+r\left(\mathfrak{I}_{2}\right), \quad r(l(\mathfrak{r}))=\mathfrak{r}
$$

hold for left ideals $\mathfrak{r}_{1}, \mathfrak{r}_{2}$ and all right ideals $\mathfrak{r}$.

(iii) $A$ is (left and right) Noetherian and left self-injective.

(iv) 1 satisfies minimum condition for right ideals and is left self-injective.

(v) A satisfies minimum condition for left ideals and is left self-injective.

Since (i) is symmetric with respect to "left" and "right," it follows that conditions obtained from (ii) $-(\mathrm{v})$ by interchanging "left" and "right" may be added to the list.

The theorem may be looked upon as a generalization of the main theorem of Ikeda [7], Ikeda-Nakayama [9]. The proof of the theorem is postponed to the next section.

Combining the theorem with Corollary 9 we obtain:

Corollary 19. If $A$ is a Noetherian Frobenius $K$-algebra over a self-injective (commutative) ring $K$ then $A$ is a quasi-Frobenius ring.

If $K$ is Noetherian and $A$ is finitely $K$-generated then also $A$ is Noetherian. Thus we obtain:

Corollary 20. $A$ Frobenius $K$-algebra $A$ over a quasi-Frobenius (commutative) ring $K$ is a quasi-Frobenius ring.

As a further application we obtain the following result in [8] (Corollary to Main Theorem) :

Corollary 21. Let $A$ be an algebra over a field $K$ with $(A: K)<\infty$. If $A$ is a quasi-Frobenius ring then either $A$ is separable (i.e. $\operatorname{dim} A=0$ ) or $\operatorname{dim} A$ $=\infty$.

Proof. Assume $\operatorname{dim} A<\infty$. Then from [4] we know that $\operatorname{dim} A$ $=1 . \operatorname{dim}_{\Lambda}(\Lambda / N)$ where $N$ is the radical of $\Lambda$. Since, by Theorem $18, A$ is left self-injective, it follows from Theorem 16 that $1 \cdot \operatorname{dim}_{\Lambda}(\Lambda / N)=0, \infty$. Thus $\operatorname{dim} \Lambda$ $=0$.

\section{§7. Proof of Theorem 18}

It is known ([3]; cf. also [1]) that a ring $A$ is left self-injective if and 
only if the following condition holds:

(a) If $\varphi: \mathfrak{l} \rightarrow \mathcal{A}$ is a $A$-homomorphism of a left ideal $\mathfrak{l}$ then there exists an element $\lambda \in A$ such that $\varphi \xi=\xi \lambda$ for all $\xi \in \mathfrak{l}$.

We shall denote by $\left(\mathrm{a}^{*}\right)$ the same condition restricted to finitely generated left ideals. We shall also consider conditions

$$
\begin{aligned}
r\left(\mathfrak{l}_{1} \cap \mathfrak{l}_{2}\right) & =r\left(\mathfrak{l}_{1}\right)+r\left(\mathfrak{l}_{2}\right), \\
r(l(\mathfrak{r})) & =\mathfrak{r}
\end{aligned}
$$

concering respectively pairs $\mathfrak{l}_{1}, \mathfrak{l}_{2}$ of left ideals of $A$ and right ideals $\mathfrak{r}$ of $A$. We shall designate by $\left(\mathrm{c}^{*}\right)$ condition $(\mathrm{c})$ restricted to finitely generated right ideals.

In a recent paper Ikeda- Nakayama [9] (cf. also Ikeda [7]) the following implications were proved

$$
(\mathrm{a}) \Rightarrow\left[(\mathrm{b}) \&\left(\mathrm{c}^{*}\right)\right] \Rightarrow\left(\mathrm{a}^{*}\right) \text {. }
$$

These will be used in the sequel; otherwise our proof of Theorem 18 will be self-contained except for basic and well known facts from the theory of rings with minimum conditions.

To prove the theorem we shall establish implications

$$
\begin{aligned}
&(\mathrm{i}) \Rightarrow(\mathrm{ii}) \Rightarrow(\mathrm{iii}) \Rightarrow(\mathrm{iv}) \Rightarrow(\mathrm{i}), \\
&(\mathrm{i}) \Rightarrow(\mathrm{v}) \Rightarrow(\mathrm{iii}) .
\end{aligned}
$$

(i) $\Rightarrow$ (ii). We only need to establish (b). Since $r$ establishes an antiisomorphism of ordered sets, lattice operations must be dualized, so that (b) holds.

(ii) $\Rightarrow$ (iii). From (I-N) we deduce $\left(\mathrm{a}^{*}\right)$. Since $A$ is left Noetherian, we obtain (a); thus $A$ is left self-injective. Since $A$ satisfies maximum condition for left ideals the condition $r(l(\mathfrak{x}))=\mathfrak{r}$ implies the minimum condition for right ideals. Thus $A$ is also right Noetherian.

(iii) $\Rightarrow(\mathrm{iv})$. From $(\mathrm{I}-\mathrm{N})$ we deduce $\left(\mathrm{c}^{*}\right)$, and since $\Lambda$ is right Noetherian, we have also (c). Since $A$ is left Noetherian it follows from $r(l(\mathfrak{r}))=\mathfrak{r}$ that $A$ satisfies also minimum condition for right ideals.

(i) $\Rightarrow(v)$. Clearly 1 satisfies minimum condition for left ideals. Further in view of the already proved implications (i) $\Rightarrow$ (ii) $\Rightarrow$ (iii), $A$ is left self-injective.

$(\mathrm{v}) \Rightarrow$ (iii). Since $A$ satisfies minimum condition for left ideals it is left 
Noetherian. From (I-N) we deduce $\left(\mathrm{c}^{*}\right)$, so that $r(l(\mathfrak{r}))=\mathfrak{r}$ for finitely generated right ideals. It follows that maximum condition holds for finitely generated right ideals. This implies that each right ideal is finitely generated i.e. that $A$ is right Noetherian.

(iv) $\Rightarrow$ (i). It suffices to establish the annihilator conditions $(\alpha)$, as left minimum condition follows automatically. From (I-N) we have $\left(c^{*}\right)$, and since $A$ is right Noetherian we have also (c), i.e. the annihilator condition for right ideals. This implies that $A$ satisfies condition

(d) If $\mathfrak{r}_{1} \equiv \mathfrak{r}_{2}$ are right ideals, then $l\left(\mathfrak{r}_{1}\right) \sqsubseteq l\left(\mathfrak{r}_{2}\right)$.

To prove the annihilator condition for left ideals, consider a (left) $A$ homomorphism $\varphi: l(r(\mathfrak{l})) \rightarrow A$ such that $\varphi(\mathfrak{l})=0$. Since $A$ is left self-injective, condition (a) holds and therefore there exists an element $\lambda \in A$ such that $\varphi \xi=\xi \lambda$ for all $\xi \in l(r(l))$. Since $\varphi(l)=0$ we have $l \lambda=0$ so that $\lambda \in r(l)$. This implies $l(r(l)) \subset l(\lambda)$ so that $\varphi=0$. We have thus proved that

$$
\operatorname{Hom}_{\Lambda}(l(r(\mathfrak{l})) / \mathfrak{l}, A)=0 .
$$

The remainder of the argument then follows from the following lemma.

LEMMA 22. Let $A$ be a ring satisfying minimum condition for right (or left) ideals in which (d) holds. Then for each non zero left A-module A we have $\operatorname{Hom}_{\mathrm{\Lambda}}(A, A) \neq 0$.

Proof. Let $N$ denote the radical of $A$. Since $N$ is nilpotent we have $A \neq N A$; set $B=A / N A$. Since $N B=0$ it follows that $B$ is completely reducible and thus admits an epimorphism $B \rightarrow C$ onto some irreducible left $A$-module $C$. We thus obtain an epimorphism $A \rightarrow C$. This reduces the proof of the lemma to the case when $A$ is irreducible.

Consider the semisimple ring $\Gamma=\Lambda / N$ which we shall regard as a twosided $A$-module. Let

$$
1=E_{1}+\ldots+E_{k}
$$

be a decomposition of the unit element in $\Lambda$ into mutually orthogonal idempotents such that

$$
\Gamma_{i}=E_{i} \Gamma=E_{i} \Gamma E_{i}=\Gamma E_{i}
$$

are the simple components of $\Gamma(i=1,2, \ldots, k)$. Then 


$$
F_{i}=N+\left(1-E_{i}\right) A=N+\Lambda\left(1-E_{i}\right)
$$

are maximal two-sided ideals in $\Lambda$. we have

$$
\begin{aligned}
l\left(F_{i}\right) & =l\left(N+\left(1-E_{i}\right) \Lambda\right)=l(N) \cap l\left(1-E_{i}\right) \\
& =l(N) \cap \Lambda E_{i}=l(N) E_{i}
\end{aligned}
$$

and similarly

$$
r\left(F_{i}\right)=E_{i} r(N) \text {. }
$$

Thus $l(N) E_{i}$ and $E_{i} r(N)$ are two-sided ideals.

If $z$ is any two-sided ideal with $z \subsetneq E_{i} r(N)$ then by condition (d)

$$
l(\mathrm{z}) \risingdotseq l\left(E_{i} r(N)\right)=l\left(r\left(F_{i}\right)\right) \supset F_{i} .
$$

Since $F_{i}$ is a maximal two-sided ideal, it follows that $l(z)=\Lambda$ i.e. $z=0$. Thus the two-sided ideal $E_{i} r(N)$ is either minimal or is zero.

Consider a composition series

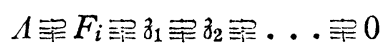

of two-sided ideals in $A$. Then by (d)

$$
0=l(\Lambda) \subsetneq l\left(F_{i}\right) \subsetneq l\left(z_{1}\right) \subsetneq l\left(z_{2}\right) \subsetneq \ldots \subsetneq A .
$$

Since the lengths of the series are equal, the latter also is a composition series and therefore $l\left(F_{i}\right)=l(N) E_{i}$ is a minimal two-sided ideal. Since $N$ is nilpotent we have $N l(N) E_{i} \subsetneq l(N) E_{i}$ and therefore $N l(N) E_{i}=0$. Thus $l(N) E_{i} \subset r(N)$, and since this holds for each $i=1,2, \ldots, k$, it follows that $l(N) \subset r(N)$. Now consider the direct sum decompositions

$$
l(N)=\sum_{i=1}^{k} l(N) E_{i}, \quad r(N)=\sum_{i=1}^{k} E_{i} r(N) .
$$

In the first, the two-sided ideals are minimal, while in the second one they are minimal or 0 . Thus $l(N) \subset r(N)$ implies that

$$
l(N)=r(N)
$$

and that each of the two-sided ideals $E_{i} r(N)$ is minimal (and not zero). (Moreover, the two decompositions coincide, i.e.there exists a permutation $\pi$ of the indices $1, \ldots, k$ such that $E_{i} r(N)=r(N) E_{\pi(i)}$ (or equivalently $E_{i} r(N) E_{\pi(i}$ $\neq 0$ )). 
Since $N E_{i} r(N)=0$ it follows that $E_{i} r(N)$ is completely reducible as a left A-module. Let $B_{i}$ be a minimal left subideal of $E_{i} r(N)$. Then $B_{i} \approx \Gamma e_{i}$ for some primitive idempotent $e_{i}$ of $\Gamma$. Since $B_{i} \subset E_{i} r(N)$ we have $B_{i}=E_{i} B_{i} \approx E_{i} \Gamma e_{i}$. Thus $\boldsymbol{e}_{i} \in \Gamma_{i}$. It follows that $A$ contains an isomorphic image of every irreducible left $\Lambda$-module. This concludes the proof.

\section{BIBLIOGRAPHY}

[1] R. Baer, Abelian groups that are direct summands of every containing abelian group, Bull. Amer. Math. Soc. 46 (1940), 800-806.

[2] R. Brauer-C. Nesbitt, On the regular representations of algebras, Proc. Nat. Acad. Sci. U.S.A. 23 (1937), 236-240.

[ 3 ] H. Cartan-S. Eilenberg, Homological Algebra, Princeton Univ. Press, 1955.

[4] S. Eilenberg, Algebras of cohomological finite dimension, Comment. Math. Helv. 28 (1954), 310-319.

[5] S. Eilenberg-M. Ikeda-T. Nakayama, On the dimension of modules and algebras, I, Nagoya Math. J. 8 (1955).

[6] G. Hochschild, Cohomology and representations of associative algebras, Duke Math. J. 14 (1947), 921-948.

[7] M. Ikeda, A characterization of quasi-Frobenius rings, Csaka Math. J. 4 (1952), 203-210.

[ 8 ] M. Ikeda-H. Nagao-T. Nakayama, Algebras with vanishing $n$-cohomology groups, Nagoya Math. J. 7 (1954).

[ 9 ] M. Ikeda-T. Nakayama, On some characteristic properties of quasi-Frobenius and regular rings, Bull. Amer. Math. Soc. 5 (1954), 15-19.

[10] H. Nagao-T. Nakayama, On the structure of $\left(M_{o}\right)$ - and $\left(M_{u}\right)$-modules, Math. Zeits. 59 (1953), 164-170.

[11] T. Nakayama, On Frobeniusean algebras, I, Ann. Math. 40 (1939), 611-633.

[12] T. Nakayama, On Frobeniusean algebras, II, Ann. Math. 42 (1941), 1-21.

[13] C. Nesbitt, On the regular representation of algebras, Ann. of Math. 39 (1938), 634-658.

Columbia University

Nagoya University 